CERN-PH-TH/2007-134

\title{
Matrix Factorizations, D-Branes and their Deformations
}

\author{
H. Jockers ${ }^{\mathrm{a}}$ and W. Lerche ${ }^{\mathrm{a}}$ \\ a Theory Division, Physics Dept. \\ CERN, 1211 Geneva 23, Switzerland
}

We review in elementary, non-technical terms the description of topological $B$-type of $D$-branes in terms of boundary Landau-Ginzburg theory, as well as some applications.

\section{INTRODUCTION}

$D$-branes (see e.g. ref. [1]) play an important rôle for understanding certain properties of string and field theories, as well as for building semirealistic models. However, practically all literature on string phenomenology deals with weakly coupled theories, where compactification radii are large and notions of classical geometry apply: e.g., supergravity solutions, branes wrapping $p$ dimensional cycles, gauge field configurations on top of branes, etc. All this corresponds just to the boundary of the parameter space, which (presumably) is a subset of measure zero of the full string parameter space.

In order to improve the understanding of how string theory behaves in the main part of its parameter space, we thus need to move away from the large radius/weak coupling regime. However, naive geometrical notions, such as a $D$-brane wrapping some $p$-dimensional cycle of a CalabiYau manifold, then start to break down. When distances become small or curvatures large, quantum corrections tend to blur notions of classical geometry, such as the dimension of a wrapped submanifold. Various physical phenomena can arise, like branes can become unstable and decay in ways that are not visible classically; orientifold planes can disintegrate; new branches in the moduli space can open up; new, non-perturbative critical points of the effective potential can develop; and contrarily, the moduli space of branes can be obstructed while classically it seems unob- structed (in other words, a non-perturbative superpotential can be generated). Recent examples of string phenomenology deep inside the "bulk" of the moduli space are given e.g., by refs. $[2,3]$.

In order to enter the bulk of the moduli space and meaningfully describe such phenomena, we need to adopt a suitable language for describing general $D$-brane configurations that goes beyond the notion of branes wrapping cycles. For topological $B$-type [4-6] $D$-branes, the proper mathematical framework is a certain enhanced, bounded derived category of coherent sheaves [7-12]; via homological mirror symmetry this maps to the category of $A$-type branes, which wrap Lagrangian cycles of the Fukaya category $[6,13]$ or coisotropic $A$-type branes [14-16].

This framework treats branes as abstract, not necessarily naive geometrical objects, but even in the geometrical, large radius limit it retains more data than the more familiar characterization of branes in terms of $K$-theory or cohomology (i.e., $R R$ charges). It thus provides a much sharper description of $D$-branes. That is, the category also contains the information about the brane locations, and other possible gauge bundle moduli. For instance, a configuration consisting of an anti$D 0$-brane located at some point $u_{1}$ of the compactification manifold, plus a $D 0$-brane located at some other point $u_{2}$, is trivial from the $K$-theory point of view, but is a non-trivial object in the categorical description as long as $u_{1} \neq u_{2}$. Obviously, this extra information is crucial for under- 
standing questions such as whether, for a given $D$ configuration, deformations are obstructed or not (i.e., what is the effective superpotential and the moduli space of its flat directions). Moreover, the language of categories is tailor-made for addressing questions about stability and bound state formation, which can be described more physically by tachyon condensation. Excellent reviews of these matters may be found in refs. [17-19].

Often physicists associate with derived categories just an abstract collection of objects (the $D$-branes) and maps (open strings) between them, and wonder what concrete physical benefit such a formal picture might provide. Indeed, by merely tracing arrows around a quiver diagram, all one obtains is a list of possible terms in the effective superpotential and these terms are usually added up with unit coefficients. However, there is much more to these maps than just being pointers between objects: in general they depend on various parameters like Calabi-Yau and brane moduli, and thus encode valuable extra information beyond mere combinatorics. Accordingly, superpotential terms derived from quiver diagrams will in general have pre-factors depending on the various moduli of the geometry, a fact that is often neglected in the physics literature.

For $B$-type topological branes, the notions of objects and morphisms (maps) can, in fact, be easily translated into a very concrete and useful language more familiar to physicists, namely the language of two-dimensional topological (twisted $N=2$ supersymmetric) boundary LandauGinzburg (LG) models [20-22]. As we will explain in more detail below, the objects, which correspond to the $D$-branes (respective boundary conditions of open string world-sheets), are represented by matrix factorizations [23,24], and the maps between them are represented by certain matrix valued, moduli dependent open-string vertex operators. That this simple physical model faithfully represents the abstract mathematical notion of the category of coherent sheaves is highly non-trivial, and has been proven recently $[25]$ to quite some generality (see also $[26,27]$ ). The key point is that the relevant category of topological $B$-type $D$-branes on a Calabi-Yau manifold described by $W=0$, is isomorphic to a certain category of matrix factorizations of $W[28,29]$. These factorizations of the from $W \mathbf{1}=\mathcal{J} \cdot \mathcal{E}$ then have a direct interpretation in terms of the boundary Landau-Ginzburg model, where $W$ figures as the bulk superpotential and $J$ as a boundary superpotential. Given a bulk Calabi-Yau geometry defined by $W=0$, the specific choice of $\mathcal{J}$ (and consequently, $\mathcal{E}$ ) encodes the specific $D$ brane geometry that the LG model describes.

A great advantage of the LG formulation, over approaches based on rational boundary CFT $[30,31]$, is that one can easily study the dependence of physical observables on moduli, and as well as on relevant deformations. In particular, it allows to quantitatively study phenomena like tachyon condensation [32], and to explicitly compute instanton corrected, effective superpotentials on world-volumes of intersecting branes [33-38]. Moreover, it allows to address global properties of branes in the Kähler moduli space [39], and more generally an extension to gauged linear $\sigma$-models allows to interpolate the brane data among different points in the Kähler moduli space [27]. Some works analyze the connection of matrix factorizations to rational boundary CFTs at the Gepner point [40-43], show the relation of matrix factorizations to homological knot invariants [44], or extend matrix factorizations to orientifold models $[45,46]$. For works covering other aspects of matrix factorizations see, for example, refs. [37, 47-56].

In these lectures, we will review some of these aspects in simple terms, leaving more complicated mathematics and technical details to the original papers. In the next section, we will recall some basic features of boundary LG theory in relation to matrix factorizations. In section 3 we will then study deformations of matrix factorizations and show how obstructions of them lead very directly to effective superpotentials. In section 4 , we will turn to discussing in more detail, as a case study, branes on the elliptic curve. This geometry is well understood both from the mathematics as well as from the physics side, and it is non-trivial enough in order to get some idea about how the 
general case works. Specifically, we will show how the bundle data $\left(r(\mathcal{E}), c_{1}(\mathcal{E}), u\right)$, that characterize a given brane, are explicitly encoded by certain properties of the matrices. Moreover we will discuss a simple example of tachyon condensation.

\section{LG MODELS AND MATRIX FAC- TORIZATIONS}

We start by briefly reviewing the bulk LandauGinzburg model; for more details about this wellknown theory, see for example [6]. It is easiest written in terms of $d=2, N=(2,2)$ superspace language as follows:

$$
S_{L G}=\int d^{2} z d \theta^{4} K\left(\Phi_{i}, \bar{\Phi}_{i}\right)+\int d^{2} z d \theta^{2} W\left(\Phi_{i}\right)+\text { c.c. }
$$

Here, $\Phi_{i}, \quad i=1, \ldots, n$ are chiral superfields, which form reduced supermultiplets that satisfy $\bar{D}_{ \pm} \Phi_{i}=0$ (in the following we will use the notation $x_{i}$ when we consider the fields as complex variables rather than superfields). Moreover, $K\left(x_{i}, \bar{x}_{i}\right)$ is the Kähler potential which is a non-holomorphic function of the LG fields, and by standard renormalization group arguments it does not play a rôle in the infrared. The superpotential $W(x)$, on the other hand, is a holomorphic function and thus protected by supersymmetry. For a quasi-homogenous superpotential, which means that it uniformly scales like $W\left(s^{q_{i}} x_{i}\right)=s W\left(x_{i}\right)$, the theory flows in the infrared to a superconformal fixed point theory that only depends on the singularity type of $W$. Given the $R$-charges $q_{i}$ of the LG fields, the central charge of that SCFT is simply given by $c=3 \sum\left(1-2 q_{i}\right)$. Note that this does certainly not specify the CFT uniquely, rather $W$ may in general contain continuous parameters on which the singularity type, and thus the CFT, depends.

The significance of $W$ is that it describes the internal compact background geometry on which the closed strings propagate. The clearest and most important geometrical interpretation is when $n=d+2$ and $\sum q_{i}=1$ : then the hypersurface defined by $W=0$ describes a Calabi-Yau $d$-fold $X_{W}$ with vanishing first Chern class. For example, in Section 4 we will focus on

$$
W\left(x_{i}, a\right)=x_{1}{ }^{3}+x_{2}{ }^{3}+x_{3}{ }^{3}-3 a x_{1} x_{2} x_{3} .
$$

The equation $W\left(x_{i}, a\right)=0$ describes the cubic elliptic curve as a sub-manifold of $\mathbb{C P}^{2}$, and $a$ parametrizes its complex structure; more details about this later.

Such a LG theory can be "twisted" by adding a background charge [6], to the effect that two of the $N=2$ supercharges turn into spin zero BRST operators. Here we will consider the " $B$ type" twist for which the BRST operators are given by $\bar{Q}_{+}$and $\bar{Q}_{-}$. Upon such a twisting, the theory becomes a topological CFT with a finite dimensional Hilbert space defined by the nontrivial cohomology of $\bar{Q}_{ \pm}$(which implies that the anti-chiral sector is dropped). More precisely, the spectrum of physical operators is given by the set of primary chiral fields, which can be represented by simple polynomials of the LG field $x$, subject to a truncation condition given by the gradient of the superpotential. In other words, the physical spectrum consists of the following polynomial ring:

$$
\mathcal{R} \cong \mathbb{C}\left[x_{i}\right] /\left[\partial_{i} W(x)=0\right]
$$

We now consider the open string version, namely the LG model (1) with superpotential $W$ on a Riemann surface with boundary, i.e., on the disk $D$. The boundary breaks the $N=(2,2)$ supersymmetry of the bulk theory to $N=2$ supersymmetry, and we will choose " $B$-type" boundary conditions [4] that are compatible with the topological twist. The surviving supercharge (rather: BRST operator) can then be taken as $Q_{B} \equiv$ $\bar{Q}_{+}+\bar{Q}_{-}$.

However, as is typical for supersymmetric Lagrangians, (1) is supersymmetry invariant only up to a total derivative. Therefore, in the presence of a boundary, supersymmetry is broken by a boundary term, which in the present context is called "Warner term" [20]. It has the following 
form:

$$
\begin{aligned}
Q_{B} \cdot & \int_{D} d^{2} z d^{2} \theta W(\Phi) \\
& =\int_{D} d^{2} z d \theta^{+} d \theta^{-}\left(\theta^{+} \partial_{+}+\theta^{-} \partial_{-}\right) W(\Phi) \\
& =\int_{\partial D} d \sigma d \theta W\left(\left.\Phi\right|_{\partial D}\right)
\end{aligned}
$$

To restore supersymmetry, one may introduce extra degrees of freedom living on the boundary, and cook up a suitable boundary potential whose supersymmetry variation cancels (4). As we will see, there is in general a large ambiguity in doing so, and the set of all consistent choices corresponds, essentially, to the set of all supersymmetric boundary conditions, or topological $B$-type $D$ branes, that are compatible with a given closed string geometry $X_{W}$.

The simplest possibility is to introduce boundary fermions $[20,57] \pi_{a}, \bar{\pi}_{a}, a=1, \ldots, k$ for some $k$, which satisfy a Clifford algebra: $\left\{\pi_{a}, \bar{\pi}_{b}\right\}=\delta_{a b}$. These fields fit into fermionic supermultiplets of the $d=1, N=2$ superalgebra, which can be compactly written as superfields of the form $\Pi_{a} \equiv\left(\pi_{a}+\theta \ell_{a}\right)$, where $\ell_{a}$ are auxiliary fields. A peculiarity of such superfields is that they are not truly chiral but satisfy $[21,22]$

$$
\bar{D} \Pi_{a}=E_{a}\left(\left.\Phi\right|_{\partial D}\right),
$$

where $E_{a}(x)$ are a priori arbitrary polynomials in $x$.

Given the fermionic boundary superfields, we can then add the following boundary superpotential term:

$$
S_{\partial}=\int_{\partial D} d \sigma d \theta \sum_{a} \Pi_{a} J_{a}\left(\left.\Phi\right|_{\partial D}\right),
$$

and one can easily check that its $Q_{B}$-variation indeed cancels the Warner term (44) iff $[23,24]$

$$
\sum_{a} J_{a}(x) E_{a}(x)=W(x) .
$$

This simple equation turns out to have important consequences! Before discussing those, let us first rewrite it by making use of the fact that the BRST operator associated with the boundary degrees of freedom can be represented as

$$
Q(x)=\pi_{a} J_{a}(x)+\bar{\pi}_{a} E_{a}(x)=\left(\begin{array}{cc}
0 & \mathcal{J}(x) \\
\mathcal{E}(x) & 0
\end{array}\right)_{2^{k} \times 2^{k}} .
$$

Here we have represented the $k$ pairs of boundary fermions by generalized, $2^{k} \times 2^{k}$ dimensional Pauli matrices. The condition (7) then turns into the following matrix equation:

$$
Q^{2}(x)=\mathcal{J}(x) \cdot \mathcal{E}(x)=\mathcal{E}(x) \cdot \mathcal{J}(x)=W(x) \mathbf{1} .
$$

This equation is, in fact, more fundamental than (77), since introducing boundary fermions is not really necessary; using appropriate superholonomy factors in the path integral [58] one can avoid fermions alltogether and write everything directly in terms of matrices. This lifts the restriction on the dimension of the matrices to coincide with the dimension of a Clifford algebra.

Eq. (9) means, essentially, that all the supersymmetric $B$-type boundary conditions (and thus, $B$-type $D$-branes) compatible with a closed string background $X_{W}$ described by $W=0$, are one-to-one to all the possible factorizations of the superpotential $W(x)$ into matrices $\mathcal{J}$ and $\mathcal{E}$ that have polynomial entries in the $x_{i}$ and otherwise are arbitrary. This is a very non-trivial mathematical statement, since we know that the $B$ type $D$-branes are described by a certain category $D^{b}\left(\operatorname{Coh}\left(X_{W}\right)\right)$ of coherent sheaves associated with $X_{W}$, which has in general a very complicated structure (in particular for Calabi-Yau threefolds). As mentioned in the introduction, this statement has been proven in [25-27] by constructing a category of matrix factorizations associated with $W$, and showing that it is equivalent to $D^{b}\left(\operatorname{Coh}\left(X_{W}\right)\right)$.

A key ingredient is that the objects $P$ in this category of matrix factorizations have a composite structure, essentially determined by the block off-diagonal pieces $\mathcal{J}$ and $\mathcal{E}$ of $Q$. These can be viewed as maps between "constituents" $p_{+}$and $p_{-}$, so that one can represent the object $P$ as a 
complex of the form 1

$$
P \cong\left(p_{+} \underset{\mathcal{J}}{\stackrel{\mathcal{E}}{\rightleftarrows}} p_{-}\right)
$$

This abstract mathematical construction (which is due to Kontsevich [28]) has a nice physical interpretation [23] which has been made rigorous in [58]: The "components" $p_{ \pm}$correspond to $D$ branes and anti- $D$-branes, and the presence of the potential $W$ forces them to condense, in the IR limit, into the $D$-brane configuration that we are interested in to describe. The maps $\mathcal{J}$ and $\mathcal{E}$, which figure as boundary potentials and whose presence is required for restoring supersymmetry (c.f., (9)), are viewed as a tachyon field configuration that effects this condensation.

This simple picture also allows us to easily understand what the geometry of the resulting $D$ brane is in terms of $\mathcal{J}$ and $\mathcal{E}$. As a toy example, consider the situation with just one LG field. Then one can write

$$
\mathcal{J}(x)=J(x)=\prod_{a=1}^{L}\left(x-u_{a}\right)
$$

which describes a system of $L D 0$ branes (located at the zeros $u_{a}$ ) that arises via condensation of a $D 2-\bar{D} 2$ system. This system does not completely annihilate because the tachyon configuration is topologically non-trivial (this is an idea originally due to Sen [59]). The first Chern class of the tachyon condensate is given by the winding number of the map $J(x)$, and thus is equal to $L$. Via the usual $K$-theory arguments, $c_{1}=L$ indeed measures the number of $D 0$-branes.

The construction is analogous for general $B$ type $D$-brane geometries. At large radius where semi-classical geometry applies, they can be thought of as vector bundles (or sheaf generalizations thereof), localized on holomorphic submanifolds of $X_{W}$. Again, the maps $\mathcal{J}$ and $\mathcal{E}$ encode certain topological data such as Chern classes of bundles and sheaves on the space $X_{W}$,

\footnotetext{
${ }^{1}$ One of the main points of the proof is to "unfold" this $\mathbb{Z}_{2}$-graded complex into a $\mathbb{Z}$-graded complex that describes a coherent sheaf in the geometrical, large radius limit. For details see [25-27].
}

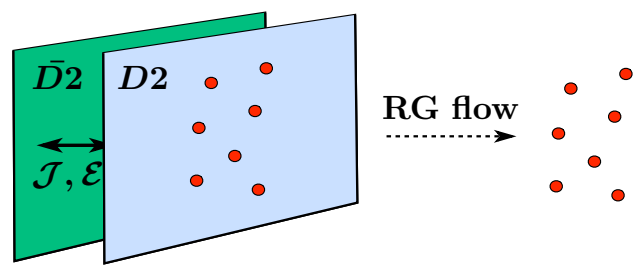

Figure 1. Tachyon condensation of a $D 2-\bar{D} 2$ system, triggered by the boundary potentials $\mathcal{J}$ and $\mathcal{E}$, leads in the infrared to a residual $D 0$-brane configuration described by the matrix factorization $\mathcal{J} \cdot \mathcal{E}=W$.

and these Chern classes directly translate to the $R R$-charges of the $D$-brane configuration that remains after tachyon condensation. We will exemplify this for the elliptic curve in Section 4.

Note, however, that this construction is more general than just $K$-theory, i.e., $R R$-charges. The maps $\mathcal{J}$ and $\mathcal{E}$ may depend also on continuous geometrical data, which correspond to deformations of the $D$-brane configuration; in the previous example these are the $D 0$ brane locations $u_{a}$ in (11). In general, two configurations which have different values of the moduli but otherwise are the same, correspond to different objects in the category, while they are indistinguishable from the $K$-theory viewpoint. For certain values, the maps may degenerate and lead to interesting physical effects, like jumps in the spectrum of physical operators. Moreover, the moduli dependence of the boundary potentials $\mathcal{J}$ and $\mathcal{E}$ trickles down to the correlation functions of the LG theory, and eventually leads to a (sometimes explicitly computable) moduli dependence of the effective action [33-38].

In order to address this kind of questions, we first of all need to define the spectrum of the physical open-string states. Again, these are viewed as maps, but now as maps between the composite objects (10). For open strings beginning and ending on the same brane $P_{a}$, which correspond to "boundary preserving" vertex operators $\Psi_{a}=\Psi_{a a}\left(u_{a}\right)$, the spectrum is simply 
given by the non-trivial BRST cohomology of $Q_{a}=Q_{a}\left(u_{a}\right)$. Mathematically speaking, they are associated with maps from the given brane onto itself, and the cohomology problem is best characterized by writing $\Psi_{a} \in \operatorname{Ext}^{s}\left(P_{a}, P_{a}\right)$, because $\Psi_{a}$ can be viewed as an extension group element at some grade $s$ (for details see e.g., $[19,60]$ ).

On the other hand, open strings that go between different branes are described by "boundary changing" operators: $\Psi_{a b}=\Psi_{a b}\left(u_{a}, u_{b}\right)$; these map between different matrix factorizations and are thus in general given by non-square matrices. For those, the proper notion of being BRST closed is:

$$
\left[Q, \Psi_{a b}\right] \equiv Q_{a} \cdot \Psi_{a b}-(-)^{s} \Psi_{a b} \cdot Q_{b}=0,
$$

and analogously for being BRST exact (the sign reflects the statistics of $\Psi_{a b}$ ). Again, the cohomology problem has a well-recognized mathematical meaning, namely in terms of extension groups $\Psi_{a b} \in \operatorname{Ext}^{s}\left(P_{a}, P_{b}\right)$ between the two $D$ branes $P_{a}$ and $P_{b}$. Such operators are localized at the intersections of the $D$-branes, and accordingly their (net) number is given by the intersection number between the branes which can be written as a topological index: $\chi_{a b}=$ $\operatorname{Tr}_{a b}(-1)^{p} \operatorname{dim} \operatorname{Ext}^{p}\left(P_{a}, P_{b}\right)$.

One often represents the open string spectrum of a brane configuration in terms of a "quiver diagram". See for example Fig. 2 which exhibits the rôle of boundary changing and boundary preserving maps, both from the space-time as well as the world-sheet perspective.

The simplest of all factorizations is the trivial factorization, where $J_{a}=1$ and $E_{a}=W$. One can easily see that there is no non-trivial open-string cohomology associated with it (not even the open-string vacuum exists), and this means that there is no $D$-brane present at all. This corresponds to the total annihilation of the $D \bar{D}$ pair due to a topologically trivial tachyon configuration. Brane configurations differing by such trivial brane-antibrane pairs are physically equivalent, and correspondingly in the mathematical formulation, the category is modded out by such "perfect complexes". For LG models this means that two factorizations of different dimen-
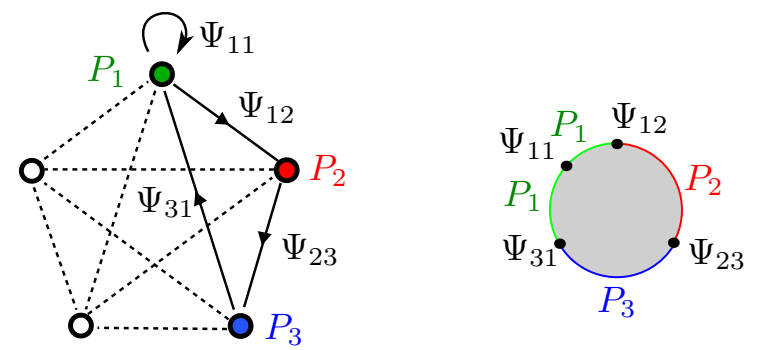

Figure 2. Left: Quiver diagram, where the nodes correspond to $D$-branes and the arrows to open strings mapping between them. Right: Disk world sheet with corresponding boundary preserving and boundary changing vertex operator insertions.

sions that are related via the addition or removal of matrix blocks of the form $(\mathbf{1}, W \mathbf{1})$, are equivalent.

What happens if we swap $\mathcal{J}$ and $\mathcal{E}$ ? While obviously the factorization of $W$ stays invariant, $\mathcal{J}$ and $\mathcal{E}$ enter differently in the LG lagrangian. It turns out that $J_{P} \rightarrow-E_{P}$ and $E_{P} \rightarrow-J_{P}$ maps a brane $P$ into its anti-brane $\bar{P}$ (in the math literature the anti-object is commonly denoted by $P[1])$. A way to see this is to show that such a $P \bar{P}$ configuration can completely annihilate under tachyon condensation.

We will outline this in the next section, but before that we introduce another feature that we are going to need, namely that of gauge symmetries. Indeed a factorization (9) is invariant under local gauge transformations of the form:

$$
\begin{aligned}
\mathcal{J}(x) & \rightarrow U_{L}(x) \mathcal{J}(x) U_{R}(x), \\
\mathcal{E}(x) & \rightarrow U_{R}^{-1}(x) \mathcal{E}(x) U_{L}^{-1}(x),
\end{aligned}
$$

for polynomial matrices $U_{L, R}(x)$ that are invertible over $\mathbb{C}\left[x_{i}\right]$. In particular, this means we can do arbitrary row and column reduction operations on $\mathcal{J}$ (respectively, $\mathcal{E}$ ), as long as we do the corresponding inverse operations on $\mathcal{E}$ (respectively, $\mathcal{J})$. This is an important tool for exhibiting the nature of a given factorization, for example a trivial block structure of the form $(\mathbf{1}, W \mathbf{1})$ may not 
be manifestly visible in a random gauge. It is therefore useful for determining the outcome of a condensation process.

\section{DEFORMATIONS}

\subsection{Bulk and boundary moduli}

One of the main advantages of the formulation of $B$-type of $D$-branes via matrix LG models over other approaches, is that it is easy to incorporate continuous deformations. It allows to study their effects on the spectrum and as well to derive the effective potential induced on them. As we will show, one can easily implement ideas of abstract deformation theory; this is useful because when deformations are obstructed and an effective potential is generated, the theory is off-shell away from the critical locus of the effective potential, and therefore we need to have a formalism that is robust enough to deal with this.

We will be interested in the following three classes of perturbations of the open string $B$ model: First, there are the usual complex structure perturbations of the bulk geometry:

$$
W(x) \longrightarrow W(x, t)=W_{0}(x)+t_{i} \phi_{i}+\ldots
$$

where the dots indicate that generically we will consider also higher orders in the perturbation.

Then there are analogous perturbations in the open string, boundary sector. Here we distinguish, as before, boundary preserving and boundary changing perturbations. The latter arise for multiple, intersecting brane configuration, and we will discuss such perturbations further below. The boundary preserving deformations

$$
Q(x) \longrightarrow Q(x, u)=Q_{0}(x)+u_{a} \Psi_{a}+\ldots
$$

are tied to a single brane and typically correspond to location moduli2 2 More precisely, from the supersymmetric GSO projection we know that the

\footnotetext{
${ }^{2}$ Note that because the actual perturbation one adds to the action is of the form $u_{a} \int d x\left\{G^{-}, \Psi_{a}\right\}$, bosonic perturbation parameters are related to fermionic vertex operators and vice versa. Only the bosonic parameters are natural physical deformations of the theory and will be considered below.
}

bulk operators and boundary preserving boundary operators have integral charges; specifically with "moduli" we refer to operators with $R$ charge $q=2$ in the bulk, or $q=1$ on the boundary, so that $t_{i}$ and $u_{\alpha}$ are dimensionless and can appear in correlation functions in a nonpolynomial way. Strictly speaking, because an effective potential will be generically generated, some or all of these parameters won't be true moduli but will be constrained.

Indeed, it is obvious that generic perturbations (1415) will spoil a given factorization and thus lead away from a supersymmetric configuration. The true moduli comprise precisely those deformations that preserve the factorization, i.e., $Q(u)^{2}=W(t)$, and these form a sub-locus of the deformation space, as is schematically shown in Fig. 3.

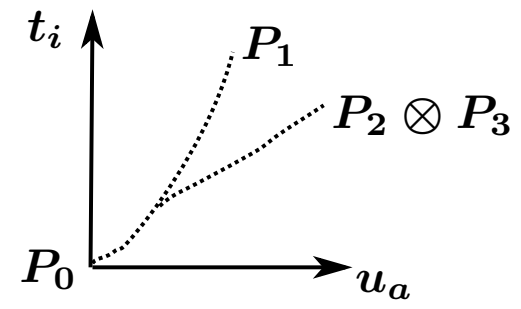

Figure 3. The supersymmetry preserving deformations form a sub-locus of the joint open/closed string deformations space. It coincides with the locus of matrix factorization which is also the critical locus of the effective superpotential. Along the sub-locus, the brane content and the open string cohomology may or may not vary, depending on whether the boundary perturbation is relevant or marginal.

As is familiar from other instances (for example from [61]), factorized geometries tend to be related to critical loci of effective superpotentials, and as we will see, this is also the case here. This has been discussed and explicitly worked out in detail $[53,62]$ for the topological minimal models as special examples, and more general discussions in the context of matrix factorizations have been 
given in refs. [33,63,64]. All this is just a specific realization of some general deformation theoretical ideas, readable accounts of which are given, for example, by [65-67]. We will give here only a crude presentation of some of the underlying ideology, and refer the reader to those papers for precise definitions and more details.

The basic principle is very simple: start with a perturbation of the BRST operator by a cohomology element as in (15), i.e., $Q=Q_{0}+Q_{1}$, with $Q_{1}=u_{a} \Psi_{a} \in \operatorname{Ext}^{1}(P, P)$. This will in general spoil factorization at the second order level, i.e., schematically:

$$
Q^{2}-W=u^{2}\{\Psi, \Psi\} \equiv: \lambda_{2}(\Psi, \Psi) .
$$

One can try to cancel this by introducing a second order correction, i.e., by adding

$$
Q_{2}=-U \lambda_{2}(\Psi, \Psi),
$$

where $U$ is a charge -1 operator that is the conjugate or "inverse" of $Q$ in the sense that $U^{2}=0$ and $\Pi=1-\{Q, U\}$ is a projector on physical states. There is an ambiguity in this definition, and we fix it by requiring a Siegel type of gauge by requiring $U \Psi=0$ on all physical states. Thus, roughly speaking, as long $\lambda_{2}(\Psi, \Psi)$ is BRST exact, we can use $Q_{2}$ to cancel the RHS of (16); however then a third order term, $m_{3}\left(\Psi^{\otimes 3}\right)$, is induced. But the procedure may be iterated and eventually yields a solution for $Q=Q_{0}+\sum_{n>0}^{\infty} Q_{n}$ given by:

$$
Q_{n}=-U \cdot \lambda_{n}\left(\Psi^{\otimes n}\right) .
$$

Here $\lambda_{n}$ are certain higher "Massey" products which are recursively defined as follows (see e.g., refs. $[67,68]$ for reviews):

$$
\begin{aligned}
& \lambda_{n}\left(\Psi_{1}, \ldots, \Psi_{n}\right)= \\
& (-)^{n-1}\left[U \lambda_{n-1}\left(\Psi_{1}, \ldots, \Psi_{n-1}\right)\right] \cdot \Psi_{n} \\
& -(-)^{n\left|\Psi_{1}\right|} \Psi_{1} \cdot U \lambda_{n-1}\left(\Psi_{2} \ldots, \Psi_{n}\right) \\
& -\sum_{\substack{l+k=n \\
k, l \geq 2}}(-)^{S}\left[U \lambda_{k}\left(\Psi_{1} \ldots, \Psi_{k}\right)\right] \cdot\left[U \lambda_{l}\left(\Psi_{k+1} \ldots, \Psi_{n}\right)\right]
\end{aligned}
$$

where $|\Psi| \in\{0,1\}$ denotes the statistics degree of a field, and $(-)^{S}$ is some complicated sign.
They form part of an $A_{\infty}$ structure that is inherent to any open string theory on the disk, and their recursive structure can be interpreted in terms of tree level Feynman diagrams associated with open string field theory [65-67]; this just reflects the inherent off-shell nature of this formalism which goes beyond conformal field theory.

However, the procedure goes through only if $Q$ can be inverted at every step - otherwise it truncates, signaling an obstruction against the given perturbation at that order. Obviously $Q$ cannot be inverted if some $\lambda_{n}$ is not BRST exact, i.e., if it is a non-trivial cohomology element 3 The condition for a perturbation to be unobstructed, and thus to preserve the matrix factorization of $W$, is

$$
\sum m_{n}\left(\Psi^{\otimes n}\right) \equiv \sum \Pi \lambda_{n}\left(\Psi^{\otimes n}\right)=0 .
$$

Taking the moduli dependence of the $\lambda_{n}$ into account, this singles out a sub-locus of the full parameter space.

In deformation theory, the equations (20) are known as generalized Maurer-Cartan equations [65-67]. They can also be interpreted as equations of motion of an open string field theory of Chern-Simons type, and within this context they can be interpreted as the critical locus of an effective superpotential. That is, from its definition as generating function of disk correlators, the latter is given by

$$
\mathcal{W}_{\mathrm{eff}}(t, u)=\sum_{n=2}^{\infty} \frac{1}{n+1}\left\langle\Psi, m_{n}\left(\Psi^{\otimes n}\right)\right\rangle,
$$

where $\langle$,$\rangle is a suitable inner product on the$ off-shell Hilbert space, and certain cyclicity properties of the $m_{n}$ are implicitly assumed to hold. Eqs. (20) are then simply the equations of motion associated with $\mathcal{W}_{\text {eff }}(t, u)$.

There is one other important aspect to note, concerning the uniqueness of the effective poten-

\footnotetext{
${ }^{3}$ Noting that the degree $s$ of $\Psi \in \operatorname{Ext}^{1}(P, P)$ equals to one, it follows that the degree of all the $\lambda_{n}$ is two, which means that abstractly speaking, the obstructions are measured by classes in $\left.\operatorname{Ext}^{2}(P, P)\right)$. The pattern continues: $\operatorname{Ext}^{3}(P, P)$ measures obstructions to obstructions, and so on $[19,60$, $69]$.
} 
tial. We already noted that the inversion of $Q$ is ambiguous, because one can always add BRST closed pieces to a BRST non-invariant one. One may fix this ambiguity by adopting the Siegel type gauge $U(\Psi)=0$ as above, but different gauges are equally well possible and lead to different results for $\mathcal{W}_{\text {eff }}(t, u)$. However, one can show [65] that the physically relevant data, i.e., the critical locus of $\mathcal{W}_{\text {eff }}(t, u)$, remain (essentially) invariant, while the directions in field space off the critical locus are ambiguous; this reflects that a given physical theory can have different off-shell completions. More precisely speaking, all those data are determined only up to field redefinitions, and that's almost the best one can hope for a topological field theory. Namely, in order to pin down the parametrization one would need to know the kinetic terms of the effective action as well, but these are not available in a TFT corresponding to $N=1$ space-time supersymmetry.

These statements apply to generic boundary perturbations, which are typically obstructed. However, in certain few circumstances, the open moduli space is flat and this can then be used to treat it similar to the closed string moduli space, i.e., define preferred flat coordinates on it. An example are $D 0$ branes on the elliptic curve which we will discuss below; the moduli space of a $D 0$ brane on some manifold $X_{W}$ is given by $X_{W}$ itself, which is flat for the torus. In contrast, $D 0$ branes on a Calabi-Yau threefold certainly don't have a flat moduli space.

\subsection{Relevant boundary deformations: tachyon condensation}

As said before, there is another class of perturbations in the open string sector, namely by boundary changing operators, $\Psi_{a b}$, acting between branes $P_{a}$ and $P_{b}$. They have quite different properties as compared to the boundary preserving moduli. In particular, their charges depend on the difference of the 'grades' of the branes they couple to [8]. These grades change continuously when we vary the Kähler moduli $[70,71]$, and therefore the charges of the open strings change too. This effect is of course not visible in the topological $B$-model, which does not de- pend on Kähler moduli. In the un-twisted physical theory, however, the Kähler moduli do matter and the masses of the open strings will depend on them. Depending on the Kähler parameters, the open strings may become tachyonic, and this is why we will denote the associated deformation parameters by $T=T_{a b}$.

Given two matrix factorizations representing branes $P_{a}$ and $P_{b}$, and a cohomologically nontrivial fermionic boundary changing operator $\Psi_{a b}$, we can form a composite matrix factorization of the form

$$
Q_{\mathrm{C}}=\left(\begin{array}{cc}
Q_{a} & T \Psi_{a b} \\
0 & Q_{b}
\end{array}\right) .
$$

It is easy to check with eq. (12) that the new matrix factorization $Q_{\mathrm{C}}$ fulfills for any value of the coupling $T$ the relation $Q_{\mathrm{C}}^{2}=W \mathbf{1}$. However, compared to deformations associated to openstring moduli there is an important difference. In the topological theory the coupling $T$ does not change the composite matrix factorization, $Q_{\mathrm{C}}$, in a continuous manner. Instead there are only two distinct choices, namely either the coupling $T$ is zero, i.e. no composite is formed, or the coupling $T$ is non-zero and a composite is formed. Different non-zero values of the coupling $T$, however, lead to gauge-equivalent composites, which all represent the very same brane configuration. Hence, in the topological theory the coupling $T$ gives only rise to a discrete choice, whereas an open-string modulus $u$ parametrizes a continuous family of inequivalent matrix factorizations.

This reminds us that we should be careful about the difference between topological tachyon condensation and tachyon condensation in the physical theory. We cannot decide within the topological sector whether a composite is stable in the underlying physical theory: this depends on whether the charge of $\Psi$ is less than one or not, i.e., whether $\Psi$ is a relevant operator or not in the physical theory. Stability of a composite is indeed a complicated concept due its dependence on the Kähler moduli $[70,71]$. Namely, in some region of the Kähler moduli space the formation of the composite is energetically favorable and the coupling $T$ acquires a non- 
zero (Kähler moduli dependent) vacuum expectation value there. Then a bound state represented by the matrix factorization $Q_{\mathrm{C}}$ is formed through tachyon condensation in the physical theory. However, in other regions of the Kähler moduli space, $T=0$ may be the energetically favored vacuum expectation value, and the composite is unstable against the decay into its constituents, $P_{a}$ and $P_{b}$. For extensive treatments of the notion of $D$-brane stability we recommend to the reader the refs. $[8,11,70-72]$.

As simplest possible example we consider, as advertized, a generic brane system given by a matrix factorization (22) for which

$Q_{a}=\left(\begin{array}{cc}0 & \mathcal{J} \\ \mathcal{E} & 0\end{array}\right), Q_{b}=-\left(\begin{array}{cc}0 & \mathcal{E} \\ \mathcal{J} & 0\end{array}\right), \Psi_{a b}=\left(\begin{array}{ll}0 & \mathbf{1} \\ \mathbf{1} & 0\end{array}\right)$.

It is easy to check that $\Psi_{a b}$ is a non-trivial cohomology element, satisfying (12) while being nonexact. It is also easy to see that for $T \neq 0$ the factorization can be brought via gauge transformations (13) to the form

$$
Q_{\mathrm{C}}=\left(\begin{array}{cccc}
0 & 0 & 0 & 1 \\
0 & 0 & -W & 0 \\
0 & -1 & 0 & 0 \\
W & 0 & 0 & 0
\end{array}\right),
$$

which, according to what we said before, represents the trivial brane configuration. This justifies a posteriori the claim that $Q_{b}$ represents the anti-brane of $Q_{a}$.

\section{BRANES ON THE ELLIPTIC CURVE}

\subsection{Setup}

In this section we will discuss the LG description of $B$-type of $D$ branes on the elliptic curve. It is the simplest model with open string moduli, but nevertheless exhibits sufficient complexity for making its detailed study quite instructive.

The moduli space of the supersymmetric $\sigma$ model with the elliptic curve as its target space is locally a product of the Kähler and the complex structure moduli space of the two-dimensional torus. A Landau-Ginzburg formulation is possible at points in the Kähler moduli space where the $\sigma$-model possesses enhanced discrete symmetries. For concreteness we choose the enhanced $\mathbb{Z}_{3}$-symmetry point in the Kähler moduli space, where the associated Landau-Ginzburg superpotential becomes

$$
W=x_{1}^{3}+x_{2}^{3}+x_{3}^{3}-3 a x_{1} x_{2} x_{3} .
$$

The variable $a$ parametrizes algebraically the complex structure moduli space of the elliptic curve and it is related the usual flat complex structure modulus $\tau$ of the two-dimensional torus by the modular invariant $j$-function:

$$
j(\tau)=\frac{3 a\left(a^{3}+8\right)}{a^{3}-1} .
$$

The matrix factorizations of the cubic curve (25) for $a=0$ have been classified in ref. [73]. To be specific, we present the two simplest matrix factorizations explicitly, however including $a$-dependence. Our first example is the factorization $Q_{L}$, consisting of the following pair of $3 \times 3$-matrices [34,35]:

$$
\begin{aligned}
\mathcal{J}_{L} & =\left(\begin{array}{lll}
\alpha_{1} x_{1} & \alpha_{3} x_{2} & \alpha_{2} x_{3} \\
\alpha_{2} x_{2} & \alpha_{1} x_{3} & \alpha_{3} x_{1} \\
\alpha_{3} x_{3} & \alpha_{2} x_{1} & \alpha_{1} x_{2}
\end{array}\right), \\
\mathcal{E}_{L} & =\frac{1}{\alpha_{1} \alpha_{2} \alpha_{3}}\left(\begin{array}{lll}
G_{11} & G_{22} & G_{33} \\
G_{23} & G_{31} & G_{12} \\
G_{32} & G_{13} & G_{21}
\end{array}\right),
\end{aligned}
$$

with the quadratic polynomials

$$
G_{j k}=\alpha_{[k+1]_{3}} \alpha_{[k+2]_{3}} x_{j}^{2}-\alpha_{k}^{2} x_{[j+1]_{3}} x_{[j+2]_{3}} .
$$

Here $[\cdot]_{3}$ indicates that the indices should be taken modulo three. The matrix pair, $\left(\mathcal{J}_{L}, \mathcal{E}_{L}\right)$, gives rise to a valid factorization of the cubic Landau-Ginzburg superpotential $W$, as long as the complex parameters $\alpha_{\ell}$ obey the cubic constraint

$$
0 \equiv \alpha_{1}^{3}+\alpha_{2}^{3}+\alpha_{3}^{3}-3 a \alpha_{1} \alpha_{2} \alpha_{3} .
$$

Since the parameters $\alpha_{\ell}$ appear only in the matrix factorization but do not enter in the bulk LandauGinzburg superpotential (25) they parametrize 
the open-string moduli space of the brane described by the matrices (27). A closer look reveals that we generate a gauge-equivalent matrix factorization by homogeneously rescaling the open-string parameters $\alpha_{\ell}$. Therefore we can view the parameters $\alpha_{\ell}$ as projective coordinates of the projective space $\mathbb{C P}^{2}$, which in addition are constrained to the hypersurface (29) $[32,35]$. Thus for the matrix factorization $Q_{L}$, we find that the open-string moduli space is just the hypersurface (29), which in turn describes a twodimensional torus 4

The second example is the matrix factorization, $Q_{S}$, which arises from the $2 \times 2$-matrix pair [35]

$$
\mathcal{J}_{S}=\left(\begin{array}{rr}
L_{1} & F_{2} \\
-L_{2} & F_{1}
\end{array}\right), \quad \mathcal{E}_{S}=\left(\begin{array}{rr}
F_{1} & -F_{2} \\
L_{2} & L_{1}
\end{array}\right)
$$

The linear polynomials, $L_{1}$ and $L_{2}$, are given by

$$
L_{1}=\alpha_{3} x_{1}-\alpha_{1} x_{3}, \quad L_{2}=\alpha_{3} x_{2}-\alpha_{2} x_{3} .
$$

If we impose again the cubic constraint (29) on the open-string parameters $\alpha_{\ell}$, then the cubic Landau-Ginzburg superpotential $W$ vanishes at the intersection locus of the two linear polynomials (31). Due to the Nullstellensatz we can then always find two quadratic polynomials, $F_{1}$ and $F_{2}$, such that

$$
W \equiv L_{1} F_{1}+L_{2} F_{2}
$$

Then the factorization (30) becomes well-defined. For the matrix factorization $Q_{S}$ we thus find again a toroidal open-string moduli space, parametrized by the projective coordinates $\alpha_{\ell}$ subject to the constraint (29).

So far we have ignored an important point. In order to really describe the supersymmetric $\sigma$ model of the elliptic curve at the enhanced symmetry point in the Kähler moduli space, we need to consider a $\mathbb{Z}_{3}$ orbifold of the Landau-Ginzburg

\footnotetext{
${ }^{4}$ For the matrix factorization $Q_{L}$ a detailed analysis reveals that due to gauge equivalences one needs to consider further identifications between the parameters $\alpha_{\ell}$. However, the resulting open-string moduli space is still a twodimensional torus with the same complex structure [32].
}

theory with cubic potential. The appropriate $\mathbb{Z}_{3}$ orbifold action on the Landau-Ginzburg fields, $x_{\ell}$, is generated by

$$
x_{\ell} \rightarrow \omega x_{\ell}, \quad \omega \equiv e^{\frac{2 \pi i}{3}} .
$$

The topological B-branes of the resulting LandauGinzburg orbifold theory are now captured in terms of $\mathbb{Z}_{3}$-equivariant matrix factorizations of the Landau-Ginzburg superpotential (25); that is to say in order to incorporate the $\mathbb{Z}_{3}$-orbifold action in the boundary sector, we need to enhance the matrix factorizations of the cubic superpotential $W$ to $\mathbb{Z}_{3}$-equivariant matrix factorizations $[32,50,51]$. This is achieved by supplementing a given factorization, defined by $Q$, with a $\mathbb{Z}_{3}$ representation, $R(k), k \in \mathbb{Z}_{3}$, such that the following equivariance condition is fulfilled:

$$
Q(x) \equiv R(k) Q\left(\omega^{k} x\right) R(-k) .
$$

Note that given an equivariant matrix factorization, $(Q, R(k))$, we immediately deduce a whole $\mathbb{Z}_{3}$-equivariant orbit of matrix factorizations distinguished by the three representations

$$
R^{a}(k)=\omega^{a k} R(k), \quad a=1,2,3 .
$$

Hence we associate to a given (indecomposable) matrix factorization of the superpotential, $W$, a whole orbit of equivariant matrix factorizations, which differ by the $\mathbb{Z}_{3}$-valued label, $a$, or more precisely by the $\mathbb{Z}_{3}$-representations, $R^{a}(k)$.

For the two matrix factorization, $Q_{L}$ and $Q_{S}$, introduced in eqs. (27) and (30), we find respectively the $\mathbb{Z}_{3}$-equivariant representations

$$
R^{L_{a}}(k)=\omega^{a k} \operatorname{diag}\left(\omega^{2 k} \mathbf{1}_{\mathbf{3} \times \mathbf{3}}, \mathbf{1}_{\mathbf{3} \times \mathbf{3}}\right),
$$

and

$$
R^{S_{a}}(k)=\omega^{a k} \operatorname{diag}\left(\mathbf{1}_{\mathbf{2} \times \mathbf{2}}, \omega^{\mathbf{k}}, \omega^{\mathbf{2 k}}\right) .
$$

In the following we denote the brane configurations associated to these equivariant matrix factorizations by the 'long' branes, $L_{a}$, and the 'short' branes, $S_{a}$. This nomenclature originates from the mirror description, where these branes wrap long and short diagonals of the A-model mirror torus [35]. 


\subsection{Bundle geometry}

At the large radius point of the Kähler moduli space, quantum corrections are suppressed and therefore classical geometry yields a good description. Thus in order to get a geometric intuitive picture of topological B-branes in the LandauGinzburg phase, we need to translate the notion of matrix factorizations into geometric notions at the large radius point (for a detailed upcoming study, see [27]). In the large radius regime topological B-branes are realized as complex vector bundles. For our purposes here the bundle picture of branes suffices, however, we should keep in mind that a more accurate description of topological B-branes at the large radius point is achieved in terms of coherent sheaves or even more precisely in terms of objects in the bounded derived category of coherent sheaves [8-12].

Our goal is now to deduce directly from the matrix factorization the associated large radius bundle data of the topological B-brane. For the elliptic curve this procedure is straightforward, since the (indecomposable) complex vector bundles on the elliptic curve have been classified a long time ago [74]. Namely such a bundle is completely characterized by its rank $r$, its first Chern number $c_{1}$, as well as by a point $u$ on the elliptic curve 5 We denote such an indecomposable vector bundle by $\mathcal{V}\left(r, c_{1}, u\right)$. The second simplification arises from the fact that at least one brane in each equivariant orbit of an indecomposable matrix factorization corresponds to a complex vector bundle in the large radius regime [32,73]. We call this vector bundle the distinguished bundle. The other branes in the same equivariant orbit give rise to vector bundles of lower rank than the distinguished bundle, and/or to more general objects such as coherent sheaves. This will become somewhat more clear in a moment.

Thus our goal is to calculate for each matrix factorization of the elliptic curve the distinguished bundle parameters $\left(r, c_{1}, u\right)$. In our two examples of factorizations given by $Q_{L}$ and $Q_{S}$, we have already encountered an open string modulus, which was encoded in the parameters $\alpha_{\ell}$ via

\footnotetext{
${ }^{5}$ Rather, on the Jacobian which happens to be isomorphic to the curve itself.
}

eq. (29). One can "uniformize" these functions in terms of theta functions (roughly by rewriting $\alpha \sim \theta(u \mid \tau)$, see $[32,35,37]$ for details), and this explicitly introduces an open string modulus $u$ which is a flat coordinate labeling a point of the (Jacobian of the) curve. Physically this point corresponds to the location of the $D 0$-brane component of the given brane configuration.

It thus remains to determine the rank $r$ and the first Chern number $c_{1}$ of the complex vector bundle. Physically these two integers represent the large radius $\mathrm{RR}$ charges of the described brane: $\left(r, c_{1}\right)=\left(N_{D 2}, N_{D 0}\right)$, up to some ambiguity to be discussed momentarily.

The key ingredient to determine the integers $\left(r, c_{1}\right)$ is to note that the image of the matrix $\mathcal{J}$, restricted to the elliptic curve $W \equiv 0$, encodes the relevant data of the distinguished bundle [32]. Furthermore, due to the factorization condition $W=\mathcal{J E}=\mathcal{E} \mathcal{J}$, one can check that on the elliptic curve $W \equiv 0$, the image of the matrix $\mathcal{J}$ coincides with the kernel of the matrix $\mathcal{E}$. This last property allows us to immediately deduce the rank $r$, of the vector bundle by simply computing the determinant of the matrix $\mathcal{E}$ :

$$
\operatorname{det} \mathcal{E} \sim W^{r} .
$$

The first Chern number of the vector bundle is just the first Chern number of the induced determinant line bundle, which is obtained by counting the number of zeros (with their multiplicities) of a global holomorphic section. Here we construct first a global holomorphic section of the image of the matrix $\mathcal{J}$, and then count the number of zeros of the induced global section of the determinant line bundle. These steps may sound a little complicated, but they essentially just generalize the simple picture of brane charges we gave in Section 2. In practice this analysis is straightforward, as will become clear by the following examples.

We consider first the distinguished bundle of the matrix factorization, $Q_{L}$, of the 'long' branes. Using eq. (29) we find

$$
\operatorname{det} \mathcal{E}_{L}=W^{2} \text {. }
$$

Hence the distinguished bundle in question is of rank two. A global section of this rank-two 
vector bundle is given by the image of the two vectors $(1,0,0)^{\mathrm{T}}$ and $(0,1,0)^{\mathrm{T}}$, namely by the first two columns $v_{1}=\left(\alpha_{1} x_{1}, \alpha_{2} x_{2}, \alpha_{3} x_{3}\right)^{\mathrm{T}}$ and $v_{2}=\left(\alpha_{3} x_{2}, \alpha_{1} x_{3}, \alpha_{2} x_{1}\right)^{\mathrm{T}}$ of the matrix $\mathcal{J}_{L}$. The zeros of the induced global section of the determinant line bundle arise at points $\left[x_{1}: x_{2}: x_{3}\right]$ in $\mathbb{C P}^{2}$, where the vectors $v_{1}$ and $v_{2}$ become linearly dependent and in addition are located on the elliptic curve $W \equiv 0$. A few steps of simple algebra reveal three such distinct points

$$
p_{s}=\left[\omega^{s} \alpha_{3}: \alpha_{1}: \omega^{-s} \alpha_{2}\right], \quad s=0,1,2 .
$$

Hence we deduce that the first Chern number of the bundle is three, and the matrix factorization $Q_{L}$ describes the distinguished bundle, $\mathcal{V}(2,3, \zeta)$. It is easy to check that two different columns of the matrix $\mathcal{J}_{L}$ lead to the same result.

Our second example is the matrix factorization (30) of the 'short' branes. Again with eq. (29) we compute $\operatorname{det} \mathcal{E}_{S}=W$, and thus we find that the factorization describes a distinguished rank-one vector bundle, i.e., a line bundle. A global section of this line bundle is given by the image of the vector $(1,0)^{\mathrm{T}}$, or in other words by the first column, $v_{1}=\left(L_{1},-L_{2}\right)^{\mathrm{T}}$, of the matrix $\mathcal{J}_{S}$. Here the determinant line bundle is obviously just the line bundle itself and hence we need to determine the number of points on the elliptic curve, where the vector $v_{1}$ vanishes. These points are given by the common zeros of the linear polynomials, $L_{1}$ and $L_{2}$, of eq. (31). One immediately finds a single zero at

$$
p=\left[\alpha_{1}: \alpha_{2}: \alpha_{3}\right] \text {. }
$$

Thus we associate to the matrix factorization $Q_{S}$ the distinguished bundle $\mathcal{V}(1,1, u)$.

Now there are a few comments in order. First of all, in the second example the attentive reader may have noticed that one obtains a different result if one constructs the global section of the image of the matrix $\mathcal{J}_{S}$ from the vector $(0,1)^{\mathrm{T}}$. For this choice one obtains the vector $v_{1}=\left(F_{1}, F_{2}\right)^{\mathrm{T}}$, and thus needs to determine the common zeros of the quadratic polynomials $F_{1}$ and $F_{2}$. Since a quadratic polynomial generically intersects the cubic polynomial $W$ in six points, the quadratics $F_{1}$ and $F_{2}$ have each six zeros on the elliptic curve.
Furthermore, using the fact that the linears, $L_{1}$ and $L_{2}$, have three zeros on the elliptic curve with one common zero at the point (41), and by carefully evaluating the relation (32) for all the zeros of the polynomials, $F_{1}, F_{2}, L_{1}$ and $L_{2}$, one finds that the polynomials $F_{1}$ and $F_{2}$ must have four common zeros. Hence from the second column of the matrix $\mathcal{J}_{S}$ one arrives at the distinguished bundle $\mathcal{V}(1,4, u)$.

This apparent discrepancy, however, has a simple physical resolution. In computing the $R R$ charges of the distinguished bundle, we implicitly specify a path in the Kähler moduli space from the Landau-Ginzburg to the large radius phase. The result depends on the choice of this path, and there is an ambiguity in the data of the distinguished bundle that is given by the monodromy about the large radius point. This monodromy amounts to tensoring the bundle data with the canonicial bundle of the ambient space $\left.\mathbb{C P}^{2}[32,39,73] 6\right]$

$$
\mathcal{V}\left(r, c_{1}, u\right) \stackrel{\text { LR monodromy }}{\longrightarrow} \mathcal{V}\left(r, c_{1} \pm 3 r, u\right)
$$

Note that we can also obtain the distinguished bundle $\mathcal{V}(1,4, u)$ from the first column of the matrix $\mathcal{J}_{S}$, by considering the global section resulting from the image of the vector $\left(x_{1}, 0\right)^{\mathrm{T}}$. Similarly we can also deduce from the matrix factorization $Q_{L}$ the distinguished bundle $\mathcal{V}(2,9, u)$, by computing the global section from the image of the matrix $\mathcal{J}_{L}$ for the vectors $\left(x_{1}, 0,0\right)^{\mathrm{T}}$ and $\left(0, x_{2}, 0\right)^{\mathrm{T}}$. Thus we can realize the large radius monodromy shifts (42) by varying the preimage sections of the matrices, $\mathcal{J} 7$

So far we have described a method to calculate on the elliptic curve the large radius $\mathrm{RR}$ charges of the brane of an indecomposable matrix factorization, which is associated to the distinguished bundle. The RR charges of the remaining branes in the equivariant orbit are then obtained by acting on them with the $\mathbb{Z}_{3}$ monodromy associated with the "Gepner point" in the Kähler moduli

\footnotetext{
${ }^{6}$ Here we refer to the large radius monodromy as seen from the gauged linear $\sigma$-model point of view (c.f. ref. [39]).

${ }^{7} \mathrm{By}$ also allowing for meromorphic preimage sections we can also obtain negative shifts for the first Chern number.
} 


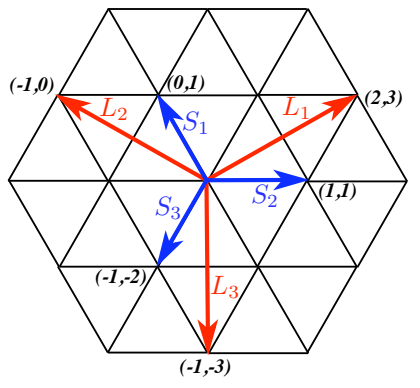

Figure 4. In the RR-charge lattice at the $\mathbb{Z}_{3^{-}}$ symmetric Landau-Ginzburg point in the Kähler moduli space, we depict the $D$-brane charges $\left(N_{D 2}, N_{D 0}\right)=\left(r, c_{1}\right)$ of the 'long' and 'short' branes $L_{a}$ and $S_{a}, a=1,2,3$. The distinguished bundles of the matrix factorizations $Q_{L}$ and $Q_{S}$, are associated to the branes $L_{1}$ and $S_{2}$, respectively.

space [39]. The resulting large radius $\mathrm{RR}$ charges of all the 'long' and 'short' branes $L_{a}$ and $S_{a}$ have been summarized in fig. 4 .

Note that the charges are not necessarily ones of nice vector bundles (i.e., of classical smooth gauge field configurations on the world-volume). For example, the $D 0$ brane formally corresponds to a "bundle" with vanishing rank but nevertheless with non-vanishing first Chern class. Clearly, no such smooth gauge field configurations exist, and this is one of the reasons why one needs to introduce mathematical notions that are more general than vector bundles, such as sheaves, in order to properly describe such objects.

In principle the described method of evaluating the bundle data also generalizes to higher dimensional target space geometries, such as the K3 surface or Calabi-Yau threefolds. There one has to determine also higher Chern numbers by counting zeros of appropriate bundles [75]. However, a closer look at a generic matrix factorization in these higher dimensional geometries reveals that it is necessary to also take into account the equivariant representations to extract the distinguished bundle data. Guided by the analysis of ref. [27], where equivariant matrix factorizations are analytically continued to the large ra- dius point in a systematic manner, it becomes clear that one needs to truncate the matrices $\mathcal{J}$ and $\mathcal{E}$ appropriately in order to determine the correct Chern numbers.

\subsection{Deformations}

\subsubsection{Tachyon condensation}

The matrix factorizations of the elliptic curve serve also as a good example to study topological tachyon condensation. Since the formation of bound states preserves the brane charges of the constituents, we obtain already selection rules for possible condensation processes by simply looking at the involved RR charges. For instance from Fig. 4 we infer that charge conservation allows the formation of the 'long' brane $L_{1}$ from the 'short' brane $S_{2}$ and the 'short' anti-brane $\bar{S}_{3}$. We will demonstrate now that such a condensation process is indeed possible.

Beforehand we point out that because a whole $\mathbb{Z}_{3}$ equivariant orbit is associated with a given matrix factorization, we need to somehow specify which precise composite out of which precise members of the two orbits under consideration we actually talk about. This extra input is provided by the choice of boundary changing operator $\Psi$ that we switch on to condense. Each channel for composite formation is characterized by a particular cohomology element, the charge of which is determined by the relative angle of the intersecting branes (in the mirror $A$-models). In other words, given two matrix factorizations, there will be a collection of boundary changing cohomology elements with different charges, and a specific choice will correspond to a tachyon consideration between specific members of the two $\mathbb{Z}_{3}$ orbits.

Thus, if we want to form a composite out of the constituents $S_{2}$ and $\bar{S}_{3}$, we first need to find the relevant fermionic boundary changing operator that triggers this particular condensation process. Evaluating the BRST cohomology (12) of the matrix factorizations $Q_{S}$ and $Q_{\bar{S}}$, one finds a fermionic operator, $\Psi_{S_{2} \bar{S}_{3}}$, which has the follow- 
ing form $[32]$

$$
\Psi_{S_{2} \bar{S}_{3}}=\left(\begin{array}{cc}
0 & \psi_{0}\left(u_{S_{2}}, u_{\bar{S}_{3}}\right) \\
\psi_{1}\left(u_{S_{2}}, u_{\bar{S}_{3}}\right) & 0
\end{array}\right) .
$$

It depends on the open-string moduli, $u_{S_{2}}$ and $u_{\bar{S}_{3}}$, of both constituents, $S_{2}$ and $\bar{S}_{3}$. The matrices, $\psi_{0}$ and $\psi_{1}$, schematically read:

$$
\begin{aligned}
& \psi_{0}\left(u_{S_{2}}, u_{\bar{S}_{3}}\right)=\left(\begin{array}{cc}
l_{5}(x) & q(x) \\
c & l_{6}(x)
\end{array}\right), \\
& \psi_{1}\left(u_{S_{2}}, u_{\bar{S}_{3}}\right)=\left(\begin{array}{ll}
l_{1}(x) & l_{2}(x) \\
l_{3}(x) & l_{4}(x)
\end{array}\right) .
\end{aligned}
$$

Here $c$ is a constant, $l_{i}(x)$ and $q(x)$ are linear and quadratic polynomials in the variable, $x$. In addition all entries have non-polynomial dependencies on the open-string moduli, $u_{S_{2}}$ and $u_{\bar{S}_{3}}$.

If we now employ the condensation formula (22), we obtain for the resulting matrix factorization, $Q_{C}$, the $4 \times 4$ matrix pair

$$
\begin{aligned}
& \mathcal{J}_{\mathrm{C}}=\left(\begin{array}{cc}
\mathcal{E}_{S}\left(u_{\bar{S}_{3}}\right) & \psi_{0}\left(u_{S_{2}}, u_{\bar{S}_{3}}\right) \\
0 & \mathcal{J}_{S}\left(u_{S_{2}}\right)
\end{array}\right), \\
& \mathcal{E}_{\mathrm{C}}=\left(\begin{array}{cc}
\mathcal{J}_{S}\left(u_{\bar{S}_{3}}\right) & \psi_{1}\left(u_{S_{2}}, u_{\bar{S}_{3}}\right) \\
0 & \mathcal{E}_{S}\left(u_{S_{2}}\right)
\end{array}\right) .
\end{aligned}
$$

A straightforward but tedious calculation reveals that this matrix pair is gauge equivalent to [32]

$$
\begin{aligned}
& \mathcal{J}_{\mathrm{C}}=\left(\begin{array}{cc}
1 & 0 \\
0 & \mathcal{J}_{L}\left(u_{S_{2}}-u_{\bar{S}_{3}}\right)
\end{array}\right), \\
& \mathcal{E}_{\mathrm{C}}=\left(\begin{array}{cc}
W & 0 \\
0 & \mathcal{E}_{L}\left(u_{S_{2}}-u_{\bar{S}_{3}}\right)
\end{array}\right) .
\end{aligned}
$$

Hence by eliminating a trivial brane-anti-brane pair, we find that the resulting matrix factorization is indeed equivalent to the matrix factorization $Q_{L}$ of the 'long' brane $L_{1}$. The appearance of the trivial brane-anti-brane pair can be traced back to the constant entry $c$ in the fermionic boundary changing operator (44), as it allows for a chain of gauge transformations that eventually makes the trivial brane-anti-brane pair manifest [32]. Moreover, we observe that the condensate

\footnotetext{
${ }^{8}$ In order to determine the equivariant labels of the boundary changing operator it is necessary to introduce an equivariant grading for the BRST cohomology elements (for details, c.f. refs. $[32,50,51]$ ).
}

depends again only on a single open string modulus given by $u_{L} \equiv u_{S_{2}}-u_{\bar{S}_{3}}$. This is in agreement with the general property of indecomposable matrix factorizations on the elliptic curve, which depend always just on a single open-string modulus [73, 74].

A known feature of the elliptic curve is the fact that any (indecomposable) matrix factorization can be obtained through successive tachyon condensation out of 'short' branes [32,73], so these serve as generators of the $D$-brane charge lattice. One certainly expects similar features to hold also for higher dimensional Calabi-Yau's.

\subsubsection{Effective superpotential}

By solving the cohomology problem for any given factorization, one can obtain explicit expressions for the moduli-dependent open string vertex operators. With those at hand, one may want to go on and compute correlation functions, for example ones that give rise to a superpotential on an intersecting brane configuration. Actually, computing correlators with more than three boundary changing operator insertions is hard, because they necessarily involve integrated insertions and there is no simple method to evaluate them except in favorable circumstances. So let's focus on three-point functions for three intersecting 'long diagonal' branes on the cubic elliptic curve for the time being [35] (see also [37]). Note that the 'long diagonal' branes intersect three times on the curve (in the $A$ model picture), so there are three boundary changing operators $\Psi_{a b}^{i}$, $i=1,2,3$, in the open string spectrum between any pair of those branes, one for each intersection point. The correlators thus have the following form:

$$
C_{i j k}=\left\langle\Psi_{12}^{i} \Psi_{23}^{j} \Psi_{31}^{k}\right\rangle
$$

and the issue is to compute their dependence on the closed and open string moduli. Matrix representatives for the $\Psi^{\prime} s$ were explicitly given in refs. $[32,35]$, and the simplest method to evaluate the correlators is to just plug these matrices into the Kapustin-Li $[23,58]$ supertrace residue 
formula:

$$
\left\langle\Psi_{12}^{i} \Psi_{23}^{j} \Psi_{31}^{k}\right\rangle=\int \frac{d^{3} x}{\prod d W(x)} \operatorname{Str}\left[d Q^{\wedge 3} \Psi_{12}^{i} \Psi_{23}^{j} \Psi_{31}^{k}\right],
$$

and evaluate it in a straightforward manner. One then obtains explicit expressions for the correlators (47), which depend on the parameters $a$ and $\alpha_{\ell}$ via the various matrices.

However the tricky part is to determine the correct normalization of the vertex operators: it is in general moduli dependent, and thus the computation done so far is seriously incomplete. Determining the normalization factors is actually the main part of the work. As it turns out [38], there exist certain differential equations that determine these normalization factors, but discussing those here would lead too far outside the scope of this lecture. At any rate, for the elliptic curve there are other, indirect arguments to infer the correct normalization [35].

After we have determined the correct normalization factors in one way or other, it is then at this point more interesting to switch to the topological $A$-model picture. This is done by employing the mirror map, which maps the $B$-model complex structure parameters $a, \alpha_{\ell}$ to flat coordinates $\tau, u$. By mirror symmetry the latter coincide with the Kähler variables of the $A$-model. As already mentioned, this map is given by (26) for the closed string modulus, and by certain theta functions for the open string moduli:

$$
\alpha_{\ell}(u, \tau)=\omega^{(\ell-1)} \Theta\left[\begin{array}{c|c}
\frac{1}{3}(1-\ell)-\frac{1}{2} & 3 u, 3 \tau \\
-\frac{1}{2} &
\end{array}\right.
$$

where $\ell=1,2,3, \omega=e^{2 \pi i / 3}, q=e^{2 \pi i \tau}$ and

$$
\Theta\left[\begin{array}{l|l}
c_{1} & u, \tau \\
c_{2} &
\end{array}\right]=\sum_{m \in \mathbb{Z}} q^{\left(m+c_{1}\right)^{2} / 2} e^{2 \pi i\left(u+c_{2}\right)\left(m+c_{1}\right)} .
$$

One can easily check that $a(\tau)$ and the $\alpha_{\ell}(u, \tau)$ as given here satisfy the cubic constraint (29).

After taking all normalization factors properly into account, the Yukawa couplings (47) then eventually come out as follows:

$$
\begin{aligned}
& C_{111}\left(\tau, u_{i}\right)=\alpha_{1}\left(u_{1}+u_{2}+u_{3}, \tau\right) \\
& C_{123}\left(\tau, u_{i}\right)=\alpha_{2}\left(u_{1}+u_{2}+u_{3}, \tau\right) \\
& C_{132}\left(\tau, u_{i}\right)=\alpha_{3}\left(u_{1}+u_{2}+u_{3}, \tau\right)
\end{aligned}
$$

(plus obvious cyclic transforms of the indices). This reproduces results first obtained in $[76,77]$. Besides potentially interesting for phenomenological model building, the $A$-model expressions (51) are interesting also for other reasons: Under mirror symmetry, the $B$ type $D 0, D 2$-branes map into $A$ type $D 1$ branes wrapping special lagrangian 1cycles, and the interpretation of the $q$ series expansion is in terms of disk instantons that span between the three intersecting $D 1$ branes in question - see Fig. 5 .

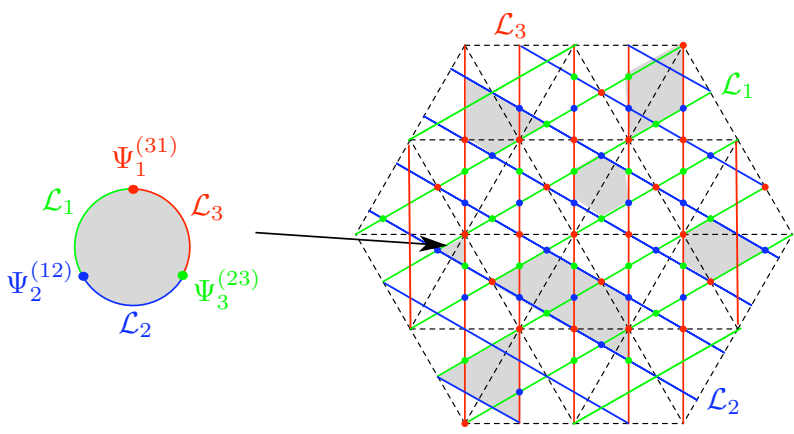

Figure 5. In the $A$-model mirror picture, the three-point function gets contributions from triangle shaped instantons that are bounded by three, pairwise intersecting $D 1$ branes. As shown, the instantons correspond to holomorphic maps from the world-sheet disk into the target space $D$-brane geometry (we drew it here on the covering space of the torus). Similarly, higher point correlators get contributions from polygonal instantons, some examples of which are shown as well. For the elliptic curve they can be evaluated directly in the $A$-model $[37,78-80]$. How to explicitly compute them for general Calabi-Yau manifolds is an open problem. 
Having computed these expansions directly from the topological $B$-model means that they represent a test of homological mirror symmetry [13, 81] (see [37] for similar computations for the quartic curve). Particularly interesting would be a generalization to higher dimensional, compact Calabi-Yau manifolds, as the issue of homological mirror symmetry becomes for those much more non-trivial. We intend to report progress on this elsewhere.

Suffice it for the time being to approach the problem of computing correlators also from a different angle, namely via the deformation theoretical method outlines in Sect. 3.1. This method is suitable for computing higher than three-point functions, but we will restrict here ourselves, for illustrative purposes, to briefly recover the previous result for the three point function in that language.

We consider the following BRST operator, which takes the brane geometry into account:

$$
Q=\left(\begin{array}{ccc}
Q_{1} & T_{12}^{i} \Psi_{12}^{i} & \\
& Q_{2} & T_{23}^{i} \Psi_{23}^{i} \\
T_{31}^{i} \Psi_{31}^{i} & & Q_{3}
\end{array}\right)
$$

We have seen in the previous section that when we switch on just a single tachyon VEV, factorization is unspoiled and thus, this deformation in unobstructed. However if we now switch on two tachyons, there is an obstruction at second order, i.e.:

$$
Q^{2}-W=T_{12}^{i} T_{23}^{j} \lambda_{2}\left(\Psi_{12}^{i}, \Psi_{23}^{j}\right)
$$

This is because in the OPE

$$
\lambda_{2}\left(\Psi_{12}^{i}, \Psi_{23}^{j}\right) \equiv \Psi_{12}^{i} \cdot \Psi_{23}^{j}=C_{i j}^{k} \Phi_{13 ; k}+\left\{Q_{13}, *\right\}
$$

there appears non-trivial bosonic cohomology elements, $\Phi_{13 ; k} \in \operatorname{Ext}^{2}\left(P_{1}, P_{3}\right)$, on the RHS. This means that the iterative procedure stops already at second order. The boundary ring structure constants, $C_{i j}^{k}$, essentially coincide with the threepoint correlators given above (again, correct normalization of all operators is essential here). To show this, it is crucial to make use of certain theta-function identities. These are of the generic form [81]

$\Theta\left(u_{1}, \tau\right) \cdot \Theta\left(u_{2}, \tau\right) \sim \sum \Theta\left(u_{1}-u_{2}, \tau\right) \cdot \Theta\left(u_{1}+u_{2}, \tau\right)$

which mirrors the structure of the OPE (54) (such identities actually encode the products in the relevant Fukaya category).

As claimed in Section 3.1, the obstruction given by the RHS of (53) determines a derivative of the effective superpotential. From (21) the latter is given (to third order) by

$$
\begin{aligned}
\mathcal{W}_{\text {eff }}(\tau, u) & \sim T_{12}^{i} T_{23}^{j}\left\langle T_{31}^{\ell} \Psi_{31}^{\ell}, \Pi \lambda_{2}\left(\Psi_{12}^{i}, \Psi_{23}^{j}\right)\right\rangle \\
& =T_{12}^{i} T_{23}^{j} T_{31}^{k} C_{i j k}(\tau, u),
\end{aligned}
$$

where we have made use of Serre duality, i.e., $\left\langle\Psi_{a b}^{\ell}, \Phi_{c d ; k}\right\rangle=\delta_{k \ell} \delta_{a d} \delta_{b c}$. Its derivative with respect to $T_{31}$ indeed yields the obstruction term in (53) (after forming a scalar expression).

\section{REFERENCES}

1. C. V. Johnson, "D-brane primer," hep-th/0007170.

2. K. Becker, M. Becker, C. Vafa, and J. Walcher, "Moduli stabilization in non-geometric backgrounds," hep-th/0611001.

3. D.-E. Diaconescu, R. Donagi, and B. Florea, "Metastable quivers in string compactifications," hep-th/0701104.

4. H. Ooguri, Y. Oz, and Z. Yin, "D-branes on Calabi-Yau spaces and their mirrors," Nucl. Phys. B477 (1996) 407-430, hep-th/9606112.

5. K. Hori, A. Iqbal, and C. Vafa, "D-branes and mirror symmetry," hep-th/0005247.

6. K. Hori, S. Katz, A. Klemm, R. Pandharipande, R. Thomas, R. Vakil, and E. Zaslow, "Mirror Symmetry," American Mathematical Society, 2003.I.

7. E. R. Sharpe, "D-branes, derived categories, and Grothendieck groups," Nucl. Phys.

B561 (1999) 433-450, hep-th/9902116.

8. M. R. Douglas, " $D$-branes, categories and N=1 supersymmetry," J. Math. Phys. 42 (2001) 2818-2843, hep-th/0011017. 
9. C. I. Lazaroiu, "Generalized complexes and string field theory," JHEP 06 (2001) 052, hep-th/0102122.

10. D.-E. Diaconescu, "Enhanced $D$-brane categories from string field theory," JHEP 06 (2001) 016, hep-th/0104200

11. P. S. Aspinwall and A. E. Lawrence, "Derived categories and zero-brane stability," JHEP 08 (2001) 004, hep-th/0104147.

12. J. Distler, H. Jockers, and H.-j. Park, " $D$-brane monodromies, derived categories and boundary linear sigma models," hep-th/0206242

13. M. Kontsevich, "Homological algebra of Mirror Symmetry," alg-geom/9411018.

14. A. Kapustin and D. Orlov, "Remarks on A-branes, mirror symmetry, and the Fukaya category," J. Geom. Phys. 48 (2003) 84, hep-th/0109098.

15. A. Kapustin and D. Orlov, "Lectures on mirror symmetry, derived categories, and $D$ branes," math.ag/0308173.

16. P. Grange and R. Minasian, "Modified pure spinors and mirror symmetry," Nucl. Phys. B732 (2006) 366-378, hep-th/0412086

17. C. I. Lazaroiu, "D-brane categories," Int. J. Mod. Phys. A18 (2003) 5299-5335, hep-th/0305095.

18. E. Sharpe, "Lectures on $D$-branes and sheaves," hep-th/0307245.

19. P. S. Aspinwall, "D-branes on Calabi-Yau manifolds," hep-th/0403166.

20. N. P. Warner, "Supersymmetry in boundary integrable models," Nucl. Phys. B450 (1995) 663-694, hep-th/9506064.

21. S. Govindarajan, T. Jayaraman, and T. Sarkar, "Worldsheet approaches to D-branes on supersymmetric cycles," Nucl. Phys. B580 (2000) 519-547, hep-th/9907131.

22. S. Govindarajan and T. Jayaraman, "On the Landau-Ginzburg description of boundary CFTs and special Lagrangian submanifolds," JHEP 07 (2000) 016, hep-th/0003242.

23. A. Kapustin and Y. Li, "D-branes in Landau-Ginzburg models and algebraic geometry," JHEP 12 (2003) 005, hep-th/0210296.

24. I. Brunner, M. Herbst, W. Lerche, and B. Scheuner, "Landau-Ginzburg realization of open string TFT," JHEP 11 (2006) 043, hep-th/0305133.

25. D. Orlov, "Derived categories of coherent sheaves and triangulated categories of singularities'," math.ag/0503632

26. P. S. Aspinwall, "The Landau-Ginzburg to Calabi-Yau dictionary for D-branes," hep-th/0610209.

27. M. Herbst, K. Hori, and D. Page to appear.

28. M. Kontsevich unpublished.

29. D. Orlov, "Triangulated categories of singularities and $D$-branes in Landau-Ginzburg models," math.ag/0302304.

30. A. Recknagel and V. Schomerus, "D-branes in Gepner models," Nucl. Phys. B531 (1998) 185-225, hep-th/9712186

31. I. Brunner, M. R. Douglas, A. E. Lawrence, and C. Römelsberger, "D-branes on the quintic," JHEP 08 (2000) 015, hep-th/9906200.

32. S. Govindarajan, H. Jockers, W. Lerche, and N. P. Warner, "Tachyon condensation on the elliptic curve," hep-th/0512208.

33. S. K. Ashok, E. Dell'Aquila, D.-E. Diaconescu, and B. Florea, "Obstructed $D$-branes in Landau-Ginzburg orbifolds," Adv. Theor. Math. Phys. 8 (2004) 427-472, hep-th/0404167.

34. K. Hori and J. Walcher, "F-term equations near Gepner points," JHEP 01 (2005) 008, hep-th/0404196.

35. I. Brunner, M. Herbst, W. Lerche, and J. Walcher, "Matrix factorizations and mirror symmetry: The cubic curve," JHEP 11 (2006) 006, hep-th/0408243

36. S. Govindarajan and H. Jockers, "Effective superpotentials for B-branes in Landau-Ginzburg models," JHEP 10 (2006) 060, hep-th/0608027

37. J. Knapp and H. Omer, "Matrix factorizations and homological mirror symmetry on the torus," hep-th/0701269

38. W. Lerche to appear.

39. H. Jockers, " $D$-brane monodromies from a 
matrix-factorization perspective," JHEP 02 (2007) 006, hep-th/0612095.

40. I. Brunner and M. R. Gaberdiel, "Matrix factorisations and permutation branes," JHEP 07 (2005) 012, hep-th/0503207.

41. I. Brunner and M. R. Gaberdiel, "The matrix factorisations of the $D$-model," J. Phys. A38 (2005) 7901-7920, hep-th/0506208.

42. H. Enger, A. Recknagel, and D. Roggenkamp, "Permutation branes and linear matrix factorisations," JHEP 01 (2006) 087, hep-th/0508053.

43. I. Brunner, M. R. Gaberdiel, and C. A. Keller, "Matrix factorisations and $D$-branes on K3," JHEP 06 (2006) 015, hep-th/0603196.

44. S. Gukov and J. Walcher, "Matrix factorizations and Kauffman homology," hep-th/0512298.

45. K. Hori and J. Walcher, "D-brane categories for orientifolds: The Landau-Ginzburg case," hep-th/0606179.

46. I. Brunner and V. Mitev, "Permutation orientifolds," hep-th/0612108.

47. A. Kapustin and Y. Li, "D-branes in topological minimal models: The Landau-Ginzburg approach," JHEP 07 (2004) 045, hep-th/0306001.

48. A. Kapustin and Y. Li, "Topological correlators in Landau-Ginzburg models with boundaries," Adv. Theor. Math. Phys. 7 (2004) 727-749, hep-th/0305136

49. M. Herbst, C.-I. Lazaroiu, and W. Lerche, "Superpotentials, $A_{\infty}$ relations and WDVV equations for open topological strings," JHEP 02 (2005) 071, hep-th/0402110.

50. S. K. Ashok, E. Dell'Aquila, and D.-E. Diaconescu, "Fractional branes in Landau-Ginzburg orbifolds," Adv. Theor. Math. Phys. 8 (2004) 461-513, hep-th/0401135.

51. J. Walcher, "Stability of Landau-Ginzburg branes," J. Math. Phys. 46 (2005) 082305, hep-th/0412274.

52. E. Dell'Aquila, " $D$-branes in toroidal orbifolds and mirror symmetry," JHEP 04 (2006) 035, hep-th/0512051.
53. J. Knapp and H. Omer, "Matrix factorizations, minimal models and massey products," JHEP 05 (2006) 064, hep-th/0604189.

54. P. S. Aspinwall, "Topological $D$-branes and commutative algebra," hep-th/0703279

55. M. Baumgartl, I. Brunner, and M. R. Gaberdiel, "D-brane superpotentials and RG flows on the quintic," arXiv:0704.2666 [hep-th]

56. I. Brunner and D. Roggenkamp, "B-type defects in Landau-Ginzburg models," arXiv:0707.0922 [hep-th]

57. S. Govindarajan and T. Jayaraman, "Boundary fermions, coherent sheaves and D-branes on Calabi-Yau manifolds," Nucl. Phys. B618 (2001) 50-80, hep-th/0104126

58. M. Herbst and C.-I. Lazaroiu, "Localization and traces in open-closed topological landauginzburg models," JHEP 05 (2005) 044, hep-th/0404184

59. A. Sen, "Tachyon condensation on the brane antibrane system," JHEP 08 (1998) 012, hep-th/9805170

60. S. Katz and E. Sharpe, "D-branes, open string vertex operators, and Ext groups," hep-th/0208104.

61. F. Cachazo and C. Vafa, "N=1 and $\mathrm{N}=2$ geometry from fluxes," hep-th/0206017.

62. M. Herbst, C.-I. Lazaroiu, and W. Lerche, " $D$-brane effective action and tachyon condensation in topological minimal models," JHEP 03 (2005) 078, hep-th/0405138.

63. K. Hori and J. Walcher, "D-branes from matrix factorizations," Comptes Rendus Physique 5 (2004) 1061-1070, hep-th/0409204.

64. D.-E. Diaconescu, A. Garcia-Raboso, R. L. Karp, and K. Sinha, "D-brane superpotentials in Calabi-Yau orientifolds (projection)," hep-th/0606180

65. C. I. Lazaroiu, "String field theory and brane superpotentials," JHEP 10 (2001) 018, hep-th/0107162.

66. H. Kajiura, "Noncommutative homotopy algebras associated with open strings," math.qa/0306332. 
67. K. Fukaya, "Deformation theory, homological algebra and mirror symmetry," Geometry and physics of branes (Como, 2001), Ser. High Energy Phys. Cosmol. Gravit (2003) 121.

68. H. Kajiura and J. Stasheff, "Open-closed homotopy algebra in mathematical physics," J. Math. Phys. 47 (2006) 023506, hep-th/0510118.

69. D. Berenstein and M. R. Douglas, "Seiberg duality for quiver gauge theories," hep-th/0207027.

70. M. R. Douglas, B. Fiol, and C. Römelsberger, "The spectrum of BPS branes on a noncompact Calabi-Yau," JHEP 09 (2005) 057, hep-th/0003263

71. P. S. Aspinwall and M. R. Douglas, "D-brane stability and monodromy," JHEP 05 (2002) 031, hep-th/0110071.

72. F. Denef, "Supergravity flows and $D$-brane stability," JHEP 08 (2000) 050, hep-th/0005049.

73. R. Laza, G. Pfister, and D. Popescu, "Maximal Cohen-Macaulay modules over the cone of an elliptic curve," Journal of Algebra 253 (2002) 209-236.

74. M. Atiyah, "Vector bundles over an elliptic curve," Proc. London Math. Soc. VII (3) (1957) 414-452.

75. We thank Nick Warner for illuminating remarks.

76. D. Cremades, L. E. Ibanez, and F. Marchesano, "Yukawa couplings in intersecting D-brane models," JHEP 07 (2003) 038, hep-th/0302105.

77. D. Cremades, L. E. Ibanez, and F. Marchesano, "Computing Yukawa couplings from magnetized extra dimensions," JHEP 05 (2004) 079, hep-th/0404229.

78. A. Polishchuk, " $A_{\infty}$-structures on an elliptic curve," Commun. Math. Phys. 247 (2004) 527-551, math.ag/0001048.

79. M. Herbst, W. Lerche, and D. Nemeschansky, "Instanton geometry and quantum $A_{\infty}$ structure on the elliptic curve," hep-th/0603085.

80. H. Kajiura, "An $A_{\infty}$-structure for lines in a plane," math.qa/0703164

81. A. Polishchuk and E. Zaslow, "Categorical mirror symmetry: The elliptic curve," $A d v$. Theor. Math. Phys. 2 (1998) 443-470, math.ag/9801119. 